\title{
Magnetization of the lunar crust
}

\author{
R. A. Carley, ${ }^{1}$ K. A. Whaler, ${ }^{1}$ M. E. Purucker, ${ }^{2}$ and J. S. Halekas ${ }^{3}$ \\ Received 26 August 2011; revised 23 June 2012; accepted 26 June 2012; published 2 August 2012.
}

[1] Magnetic fields measured by the satellite Lunar Prospector show large scale features resulting from remanently magnetized crust. Vector data synthesized at satellite altitude from a spherical harmonic model of the lunar crustal field, and the radial component of the magnetometer data, have been used to produce spatially continuous global magnetization models for the lunar crust. The magnetization is expressed in terms of localized basis functions, with a magnetization solution selected having the smallest root-mean square magnetization for a given fit to the data, controlled by a damping parameter. Suites of magnetization models for layers with thicknesses between 10 and $50 \mathrm{~km}$ are able to reproduce much of the input data, with global misfits of less than $0.5 \mathrm{nT}$ (within the uncertainties of the data), and some surface field estimates. The magnetization distributions show robust magnitudes for a range of model thicknesses and damping parameters, however the magnetization direction is unconstrained. These global models suggest that magnetized sources of the lunar crust can be represented by a $30 \mathrm{~km}$ thick magnetized layer. Average magnetization values in magnetized regions are $30-40 \mathrm{~mA} / \mathrm{m}$, similar to the measured magnetizations of the Apollo samples and significantly weaker than crustal magnetizations for Mars and the Earth. These are the first global magnetization models for the Moon, providing lower bounds on the magnitude of lunar crustal magnetization in the absence of multiple sample returns, and can be used to predict the crustal contribution to the lunar magnetic field at a particular location.

Citation: Carley, R. A., K. A. Whaler, M. E. Purucker, and J. S. Halekas (2012), Magnetization of the lunar crust, J. Geophys. Res., 117, E08001, doi:10.1029/2011JE003944.

\section{Introduction}

[2] Since the Apollo missions it has been known that while the Moon possesses no global magnetic field, it has regions of weak magnetic field resulting from areas of the crust that are remanently magnetized. The NASA orbiting satellite Lunar Prospector [Binder, 1998] made measurements of the lunar magnetic field with a magnetometer (MAG), and made estimates of the surface field magnitude with an Electron Reflectometer (ER), providing the first global maps of the lunar magnetic field [Richmond and Hood, 2008; Purucker, 2008; Mitchell et al., 2008].

[3] The main features of the lunar crustal field are their association with the antipodes of the youngest and similarly aged large impact basins [Lin et al., 1988; Mitchell et al., 2008; Halekas et al., 2001; Hood et al., 2001], and the presence of strong isolated anomalies. While the antipodes of the young impact basins show very strong magnetic fields, the large mare-filled impact basins themselves, and other lunar

\footnotetext{
${ }^{1}$ School of GeoSciences, University of Edinburgh, Edinburgh, UK.

${ }^{2}$ SGT at Planetary Geodynamics Laboratory, Goddard Space Flight Center, Greenbelt, Maryland, USA.

${ }^{3}$ Space Sciences Laboratory, University of California, Berkeley, California, USA.

Corresponding author: R. A. Carley, School of GeoSciences, University of Edinburgh, Edinburgh EH9 3JW, UK. (r.a.carley@cantab.net)

(C)2012. American Geophysical Union. All Rights Reserved. 0148-0227/12/2011JE003944
}

craters, show magnetic lows compared with their surroundings [Halekas et al., 2002], with the exception of some older (pre-Nectarian/Nectarian), larger $(>50 \mathrm{~km}$ diameter) impact basins which show weak central magnetic fields [Halekas et al., 2003; Purucker et al., 2008; Hood, 2011]. Many of the strong anomalies, such as the Reiner Gamma anomaly, are associated with regions of high albedo [Richmond et al., 2003], often swirl-like in nature [e.g., Hood and Schubert, 1980], suggested to result from differential weathering (optical maturation) of the lunar regolith due to deflection of the solar wind particles by the magnetic anomaly.

[4] The large scale $(>500 \mathrm{~km})$ magnetic anomalies measured by satellites imply the presence of relatively strong and coherent sources of remanent magnetization. However, surface magnetometers showed crustal fields with great variety in strength and direction over distances of only a few $\mathrm{km}$, suggesting they are associated with localized sources of magnetization close to the surface [Dyal et al., 1970]. Large natural remanent magnetizations of the returned Apollo samples [Runcorn et al., 1970] showed that the main carriers for this remanent magnetization were metallic iron particles [Fuller, 1974]. Iron can be derived from the bedrock or can be added to lunar soils and breccias during impacts [e.g., Housley et al., 1973], and the strongest fields are in regions linked with impact ejecta [Strangway et al., 1973; Dyal et al., 1974].

[5] Interpretation of lunar magnetism relies on the determination of the distribution, magnitude and direction of the 
sources of magnetization. In the absence of extensive sampling, or information about possible source locations and dimensions, this is made possible with magnetization models produced from the observed magnetic field data. Inverse models that do not require assumptions about the magnetization direction are particularly important for the lunar case where directions cannot be assumed.

[6] The origins of the magnetic fields that resulted in this remanent magnetization, and the mechanisms by which the remanent magnetization was acquired, are still under discussion. The main contenders for the lunar paleofields are an ancient lunar dynamo, and processes occurring on the Moon as a result of impacts [e.g., Collinson, 1993], with magnetization features resulting from excavation, heating and cooling in the presence of a field, or shock magnetization (or demagnetization). Given the highly cratered surface of the Moon, and the association of many of the lunar magnetic anomalies with impacts or impact ejecta, it is likely that impact processes are an important feature of lunar magnetism.

[7] The strong remanent magnetization measured in the Apollo samples [e.g., Fuller and Cisowski, 1987; GarrickBethell et al., 2009] and the central basin anomalies might suggest the existence of a past lunar dynamo [e.g., Hood, 2011]. While several mechanisms have been proposed for dynamo generation [e.g., Stegman et al., 2003; Le Bars et al., 2011; Dwyer et al., 2011], magnetization in a dynamo field may not be able to explain all of the features of the lunar crustal fields. The antipodal relationship between impact basins and magnetic anomalies has been explained by the focusing of impact generated plasmas and amplification of an ambient magnetic field at the antipode [Hood and Vickery, 1984; Hood, 1987; Hood and Huang, 1991; Hood and Artemieva, 2008], with magnetization occurring through low pressure shocks [Gattacceca et al., 2010]. Hypervelocity impacts are also capable of producing large transient magnetic fields [Srnka, 1977; Crawford and Schultz, 1988].

[8] Whatever the magnetic fields responsible for producing the remanent crustal fields, they must be able to explain both the presence and the absence of crustal fields in different locations on the Moon and in rocks of different ages. Understanding the origins of the lunar magnetic fields has relevance to the understanding of terrestrial planets and their evolution, with implications for the magnetization of smaller bodies, either through dynamo generation or other means of magnetic field generation or enhancement.

[9] In this study, we follow the method of Whaler and Langel [1996] and Whaler and Purucker [2005] to produce a spatially continuous magnetization model for the lunar crust as a global layer with constant thickness, using inverse techniques based on those developed for terrestrial and Martian satellite data sets. As well as being able to describe the observed magnetic fields, magnetization models should be able to provide information on the properties of the magnetized sources, namely the magnetization contrast, source thickness and dimensions.

\section{Data}

[10] Crustal magnetization models require crustal magnetic field measurements, which must be isolated from the total magnetic field measured by the MAG. The magnetization models presented here are produced using the radial, theta and phi components of the magnetic field synthesized from a degree 150 spherical harmonic model of the crustal field [Purucker, 2008], and also the altitude normalized (to $30 \mathrm{~km}$, using an equivalent source dipole technique) radial component of the crustal magnetic field isolated by Purucker [2008]. These data utilize the low altitude $(<66 \mathrm{~km}$, average $30 \mathrm{~km})$, high inclination phase of the MAG data from the lunar wake and magnetotail times where the solar wind plasma densities are low and external field variations minimal. Altitude normalized, rather than original MAG data, were used because the normalization process was part of isolating the crustal field from remaining smaller scale external fields, and MAG data at the original altitudes (not normalized or modeled) are noisy. Description of the isolation of the crustal field from the original MAG data and the production of the spherical harmonic model are found in Purucker [2008], along with links to the data sets.

[11] Since the production of these models, a new spherical harmonic model for the lunar crustal field has been produced by Purucker and Nicholas [2010], which supersedes the maps of Purucker [2008]. However, the degree by degree correlation between the models of Purucker [2008] and Purucker and Nicholas [2010] is higher than 0.75 for degrees 15-170, and the magnetic field predictions show a linear correlation coefficient of 0.9 . While an error was identified in the isolation process of Purucker [2008], reducing the magnitude of the radial component, this would not make a significant difference to the models described here, and the inferences from them.

[12] Spherical harmonic terms up to degree 150 (considered robust [Purucker, 2008]) were used to reproduce the vector components of the magnetic field at $30 \mathrm{~km}$ altitude (defined above the surface of a spherical Moon of radius $1737.1 \mathrm{~km})$ at evenly distributed points. Data locations were assigned using a 'polar coordinate subdivision' [Katanforoush and Shahshahani, 2003], placing equally spaced points on equally spaced $\left(1^{\circ}\right)$ latitudes, resulting in 35812 data locations with mean spacing $\sim 1.1^{\circ}$. This gives crustal field values up to $\pm 18 \mathrm{nT}$, which should be considered a minimum estimate of the strength of the field at a given location, due to the smoothing out of smaller scale, higher magnitude features in the spherical harmonic representation. The overall noise level of the synthesized and processed MAG data is dominated by the external magnetic field environment, and amounts to $\sim 0.5 \mathrm{nT}$.

[13] The radial field component of the crustal field isolated by Purucker [2008] retains higher amplitude unsmoothed information from each satellite pass in the lunar wake and magnetotail, and though altitude normalized, contains discontinuities due to upward or downward continuation from the initial data altitude. A sub-set of these data was selected at points closest to the locations of a polar coordinate subdivided grid with $\sim 1.1^{\circ}$ mean spacing. Subsequently, points selected closest to the same grid shifted by $0.25^{\circ}$ in latitude and longitude were used to assess the stability of the magnetization solutions with respect to the input data, and provide an upper bound on the uncertainties in the deduced magnetizations.

[14] The scalar estimates of the surface field from the Electron Reflectometer are not used to produce these 
models, but the (equal angle) $1^{\circ}$ binned ER data are compared to the surface field predictions from these models, as an independent validation of the ability of these models to reproduce magnetic field data.

\section{Modeling}

[15] The inverse modeling method follows that of Whaler and Purucker [2005], using a minimum norm strategy to find a magnetization solution with the minimum root-mean square (RMS) magnetization amplitude for a global layer with constant thickness. This avoids magnetizations stronger than absolutely required to fit to the data. An alternative norm to minimize would be a measure of the spatial gradient of the solution [e.g., Constable et al., 1987]. However, this would smooth out any rapid changes in magnetization expected from sharp geological boundaries, and edges of features associated with magnetization or demagnetization processes.

[16] While we do not expect a magnetized layer of uniform thickness, given the lack of information about possible source locations and dimensions for the Moon (and the lack of clear correlations between magnetic anomalies with Clementine gravity and topography data [Frey et al., 2002]), a magnetized layer with constant thickness is not an unreasonable assumption. For the Earth, the whole of the crust down to the Curie isotherm or the crust/mantle interface (whichever is shallower) can be considered to be magnetized in the presence of the global magnetic field. However, for the Moon the actual region of the crust which is magnetized will depend on the extent of the magnetizing fields, and the types of sources.

[17] The thickness of this global layer, extending from the surface, was varied from $5 \mathrm{~km}$ to $60 \mathrm{~km}$, to reflect the possible magnetization thicknesses for the Moon; magnetization may exist as a thin layer of impact ejecta or mare basalt, or within the entire thickness of the crust, estimated from Lunar Prospector and Clementine gravity and topography data to be $49 \pm 16 \mathrm{~km}$ on average [Wieczorek et al., 2006]. Halekas et al. [2002] used the demagnetization signatures of large $(>50 \mathrm{~km})$ craters to place an upper limit on the thickness of the lunar magnetized layer at $\sim 50 \mathrm{~km}$ [Halekas et al., 2002]. Following Voorhies et al. [2002], the global magnetic crustal thickness was estimated from the spectral content of the lunar crustal field to be $25-30 \mathrm{~km}$ (see Appendix A). Applied to satellite data, our modeling technique is not expected to be able to resolve depth variations of magnetization. However, since we used data normalized to, or synthesized at, only $30 \mathrm{~km}$ altitude, varying the thickness of the magnetized layer may indicate the most appropriate average global thickness of the magnetized layer, which may in turn offer some constraints on the types of sources (e.g., volcanic or ejecta) or on the magnetizing field.

[18] The continuous magnetization vector $\mathbf{M}(\mathbf{s})$, for three components of magnetization is expressed as a linear combination of Green's functions $\mathbf{G}\left(\mathbf{s}, \mathbf{r}_{i}\right)$, with expansion coefficients $\alpha_{i}$, relating the magnetization at a location $\mathbf{s}$ within a layer to $N$ magnetic field observations at data locations $\mathbf{r}_{i}$ [Parker et al., 1987; Shure et al., 1982]:

$$
\mathbf{M}(\mathbf{s})=\sum_{i=1}^{N} \alpha_{i} \mathbf{G}\left(\mathbf{s}, \mathbf{r}_{i}\right)
$$

The Green's function relating magnetization to the $p$ th component of the observed magnetic field is given by

$$
\mathbf{G}^{(p)}\left(\mathbf{r}_{i}, \mathbf{s}\right)=-\frac{\mu_{\mathrm{o}}}{4 \pi} \hat{\mathbf{I}}_{i}^{(p)} \cdot \nabla_{r_{i}} \nabla_{s} \frac{1}{\left|\mathbf{r}_{i}-\mathbf{s}\right|}
$$

with $\hat{\mathbf{I}}_{i}^{(p)}$ the unit vector in the direction of the local orthogonal components. Application of the gradient operators with respect to positions $\mathbf{r}$ and $\mathbf{s}$ give basis functions (and hence magnetization) that decrease with depth into the magnetized layer, inversely proportional to $\left|\mathbf{r}_{i}-\mathbf{s}\right|^{3}$.

[19] The set of magnetic field observations $\mathbf{B}$, of single or multiple components, can be represented as a system of linear equations:

$$
\mathbf{B}=\boldsymbol{\Gamma} \alpha
$$

where $\boldsymbol{\Gamma}$ is the 'Gram matrix' containing the volume integrals of all the possible pairs of dot products of the basis functions for the data points ordered in vector $\mathbf{B}$, and $\boldsymbol{\alpha}$ is the vector of coefficients of the basis functions given in (1). A solution for $\boldsymbol{\alpha}$ with minimum magnetization amplitude for a given fit to the data is obtained from [Shure et al., 1982]

$$
\mathbf{B}=(\boldsymbol{\Gamma}+\lambda \mathbf{I}) \boldsymbol{\alpha}
$$

where $\mathbf{I}$ is the identity matrix and $\lambda$, known as a damping parameter, controls the relative importance of the fit to the data versus the amplitude of the magnetization solution. As the damping parameter is increased, the magnitude of magnetization, and any features of the model relating to noise in the input data, are expected to be reduced at the expense of the fit to the data.

[20] The system of equations (4) is too large to be solved directly for the data sets considered here. For a magnetization distribution confined to a layer of constant thickness, the forms of the elements of the Gram matrix, given in Jackson [1990] and Whaler and Langel [1996], can be expressed as functions of the cosine of the angular separation of data points. For large angular separations, they become very small and the Gram matrix can be made sparse by treating as nonzero only matrix elements with absolute values above a stated threshold. Following the method of Whaler and Purucker [2005], a solution for the expansion coefficients can be found using an iterative conjugate gradient method for sparse matrix systems [Purucker et al., 1996], with Jacobi scaling. The solution was deemed to have converged when equation (4) was satisfied to a tolerance of $<10^{-10}$, within 500 iterations. Failure to converge within 500 iterations would indicate an ill-conditioned system, requiring a larger damping parameter.

[21] Alongside the solution vector $\boldsymbol{\alpha}$, the sum of squares of residuals (SSR) was calculated from [Shure et al., 1982]

$$
S S R=\lambda^{2} \boldsymbol{\alpha}^{T} \boldsymbol{\alpha}
$$

(more usefully expressed as the RMS misfit), and the solution norm $\|\mathbf{M}\|$, representing the RMS magnetization 
Table 1. Model Outputs for a $30 \mathrm{~km}$ Thick Magnetized Layer ${ }^{\mathrm{a}}$

\begin{tabular}{|c|c|c|c|c|c|c|c|}
\hline \multirow[b]{2}{*}{ Damp. $\lambda$} & \multirow[b]{2}{*}{$\operatorname{SSR}\left(n T^{2}\right)$} & \multirow[b]{2}{*}{$\operatorname{Norm}(\mathrm{A} / \mathrm{m})$} & \multirow[b]{2}{*}{ Misfit (nT) } & \multirow[b]{2}{*}{ Iterations } & \multicolumn{3}{|c|}{ Correlation Coefficients } \\
\hline & & & & & $\mathrm{Br}$ & $\mathrm{Bt}$ & $\mathrm{Bp}$ \\
\hline 0 & 0 & 2228 & 0 & 30 & 1 & 1 & 1 \\
\hline 100 & 0.300 & 2223 & 0.0017 & 30 & 1 & 1 & 1 \\
\hline 1000 & 27.44 & 2175 & 0.0160 & 26 & 1 & $>0.999$ & $>0.999$ \\
\hline 5000 & 500.2 & 2010 & 0.0682 & 22 & 0.999 & 0.999 & 0.998 \\
\hline 10000 & 1536 & 1858 & 0.1196 & 18 & 0.998 & 0.997 & 0.996 \\
\hline 50000 & 13090 & 1246 & 0.3490 & 11 & 0.985 & 0.983 & 0.98 \\
\hline 100000 & 25409 & 909.4 & 0.4863 & 8 & 0.976 & 0.974 & 0.969 \\
\hline
\end{tabular}

${ }^{a}$ Models produced from three components of the magnetic field synthesized from the spherical harmonic model of Purucker [2008]. Linear correlation coefficients [e.g., Press et al., 1992] are between the predicted and input data.

amplitude for the entire volume of the magnetized layer, was also calculated:

$$
\|\mathbf{M}\|=\sqrt{\int_{V} \mathbf{M} \cdot \mathbf{M} d V}=\sqrt{\boldsymbol{\alpha}^{T} \boldsymbol{\Gamma} \boldsymbol{\alpha}}
$$

The three components of the continuous distribution of magnetization obtained by substituting the solution for $\boldsymbol{\alpha}$ from (4) into (1) were evaluated on grids with $0.5^{\circ}$ and $0.25^{\circ}$ spacing, at a specified depth within the magnetized layer. To allow a more meaningful comparison of models with different layer thicknesses, we calculated the vertically integrated magnetization (VIM, in units $\mathrm{kA}$ ).

[22] Ideally the crustal magnetization models would be able to adequately account for both the MAG data at altitude and the ER data at the surface. The magnetization solutions were also used to predict the magnetic field at the surface locations of the $1^{\circ}$ binned ER data for comparison. However, the surface fields predicted from the magnetization model are not expected to agree in magnitude with the ER data due to the differences in the sensitivity of the MAG and the ER technique. The ability of the magnetization model to predict the ER data acts as a test for the presence of annihilators of the magnetic inverse problem [Maus and Haak, 2003], a source of non-uniqueness [Runcorn, 1975]. Magnetic annihilators contribute to the solution without producing magnetic fields at the observation locations; however, they may produce nonzero magnetic fields at other locations.

\section{Results}

[23] Solutions for models from three components of the synthesized magnetic field with a range of layer thicknesses, and damping parameters between 0 and 100,000, converged within 80 iterations or fewer. Fewer iterations were required for models with larger damping parameters. Models from the radial field component data required higher damping parameters than those used for the models from the synthesized data.

[24] The robustness of the solution to the number of nonzero elements in the sparse Gram matrix was tested, and for a $30 \mathrm{~km}$ thick layer, threshold values from 0.1 to 10 retained from $9.18 \%$ to $0.988 \%$ of the Gram matrix elements respectively with very little difference (to within less than $0.05 \%$ ) in the SSR and the solution norm for each of these models. A threshold value of 1.5 was used for the models with layer thickness from $8 \mathrm{~km}$ to $60 \mathrm{~km}$, retaining between
$1.3 \%$ and $3.7 \%$ of the Gram matrix elements. Thinner layers needed lower threshold limits to converge and are deemed less reliable due to potential instabilities in the computations.

[25] Model solutions from three components of the synthesized magnetic field fit the input data to within the estimated noise level (see Table 1 for a $30 \mathrm{~km}$ thick layer), with globally averaged misfits of $<0.5 \mathrm{nT}$, and high global linear correlation coefficients $(>0.94)$ between the predicted and input synthesized fields. Solutions from the radial field component data resulted in higher misfits due to the greater range in the input data values, so while mentioned here are not discussed in as much detail as the models from the synthesized data.

[26] While a convergent solution was found with zero misfit when no damping was used with the three components of the synthesized field, such a model is likely to contain features which relate to noise in the data. Damping parameters between $\sim 10,000$ and 50,000 (comparable to the magnitudes of the diagonals of the Gram matrix) give models with similar fits to the data having lower RMS magnetizations, so represent models which optimally minimize both the residuals and the magnetization.

[27] Discussions of the possible thickness of the magnetized layer are based on model solutions from the three components of synthesized data, for layers with thicknesses of $10 \mathrm{~km}, 30 \mathrm{~km}$ and $50 \mathrm{~km}$ with the same misfit (of $\sim 0.2 \mathrm{nT}$ ). The component and total magnetization solutions for these models, calculated at the surface, can be found in the auxiliary material. ${ }^{1}$ These models have the same number of iterations despite their different damping parameters and thicknesses, and give very similar, and high, correlation coefficients and similar VIM (Table 2). The total magnetizations for these models at the top and bottom of the layer are shown in Figure 1, showing that the pattern (and direction) of magnetization remains constant with depth but decreases in amplitude. The $10 \mathrm{~km}$ thick layer requires the lowest damping parameter to give the same fit to the data as the $30 \mathrm{~km}$ and $50 \mathrm{~km}$ thick layers, and shows a higher solution norm for the same damping parameter. The 'intermediate thickness' model, of $30 \mathrm{~km}$, has the smallest misfit and smallest RMS magnetizations for most damping parameters: models with thicker and thinner layers require a larger RMS magnetization to give the same misfit as that for the $30 \mathrm{~km}$ thick layer model.

\footnotetext{
${ }^{1}$ Auxiliary materials are available at $\mathrm{ftp} / / \mathrm{ftp}$. agu.org/apend/je/ $2011 \mathrm{je} 003944$
} 
Table 2. Model Outputs, Including Fit to the ER Data, for Selected Models With Different Thickness Magnetized Layers ${ }^{\mathrm{a}}$

\begin{tabular}{cccccccccc}
\hline & & & & & & & \multicolumn{2}{c}{ Correlation Coefficients } \\
\cline { 7 - 11 } Thick. $(\mathrm{km})$ & Damp. $\lambda$ & $\begin{array}{c}\text { SSR } \\
\left(\mathrm{nT}^{2}\right)\end{array}$ & $\begin{array}{c}\text { Norm } \\
(\mathrm{A} / \mathrm{m})\end{array}$ & $\begin{array}{c}\text { Misfit } \\
(\mathrm{nT})\end{array}$ & $\begin{array}{c}\text { ER Misfit } \\
(\mathrm{nT})\end{array}$ & $\begin{array}{c}\text { VIM } \\
(\mathrm{kA})\end{array}$ & $\mathrm{Br}$ & $\mathrm{Bt}$ & $\mathrm{Bp}$ \\
\hline 10 & 15000 & 4581 & 1991 & 0.207 & 16.5 & 0.094 & 0.997 & 0.997 & 0.996 \\
30 & 20000 & 4227 & 1638 & 0.198 & 17.0 & 0.139 & 0.994 & 0.993 & 0.991 \\
50 & 16000 & 4164 & 1761 & 0.197 & 17.2 & 0.208 & 0.993 & 0.989 & 0.986 \\
\hline
\end{tabular}

${ }^{a}$ Models produced from the three components of the magnetic field synthesized from the spherical harmonic model of Purucker [2008]. Linear correlation coefficients [e.g., Press et al., 1992] are between the predicted and input data.

[28] For magnetization confined to layers with thicknesses from 10 to $50 \mathrm{~km}$, magnetization amplitudes of at least $75 \mathrm{~mA} / \mathrm{m}$ are required to produce the strongest observed magnetic fields. The average surface magnetization is very weak at $5.4 \mathrm{~mA} / \mathrm{m}$, while the crustal average is $4.9 \mathrm{~mA} / \mathrm{m}$ (for the $30 \mathrm{~km}$ thick layer), reflecting a modest reduction in magnetization amplitude with depth. Average values in magnetized regions are $\sim 30-40 \mathrm{~mA} / \mathrm{m}$, while maximum values in the most strongly magnetized areas such as the Reiner Gamma region are up to $120 \mathrm{~mA} / \mathrm{m}$.

[29] Figure 2 shows the three component magnetizations calculated at the surface for a model from the synthesized data with a $30 \mathrm{~km}$ thick magnetized layer, selected to have misfit $0.2 \mathrm{nT}$. The magnetizations in the radial direction are the strongest, and the magnetizations in the theta direction are the weakest. The magnetizations vary in direction over small regions, mirroring the directions and relative amplitudes of the vector components of the crustal field. The magnetization features seen in this model are present in those for all thicknesses and damping parameters, but with different amplitudes.
[30] Magnetization solutions from the radial field component on the global scale are almost identical to the solutions obtained from the synthesized data in Figures 1 and 2, and are hence not shown separately. However, on a smaller scale, the solutions from the radial data show finer scale features at the edges of magnetized regions. Magnetization distributions for models from the radial data on shifted grids, while having the same solution norm and SSR (to within $1 \%$ ), showed visible differences to each other in the finer scale detail of the patterns of magnetization, highlighting an instability with respect to the inversion of unsmoothed data.

[31] Predictions of the magnetic field observations (using equation (3)) from both the models from the synthetic data and the radial data show that the pattern of most of the small scale and strong features of the field are reproduced, but with reduced magnitudes. The absolute residuals, defined as $\mid$ data $|-|$ predictions $\mid$, shown in Figure 3 for the model from the synthetic data, show this under-prediction of the data as positive residuals, and also over-prediction (negative residuals) around the edges of the strongest anomalies. The largest residuals, of up to $\pm 5 \mathrm{nT}$ (higher for the more noisy a

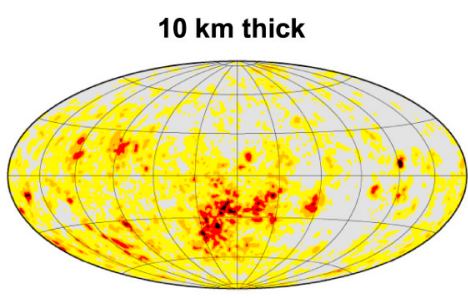

$180^{\circ} \mathrm{W}$
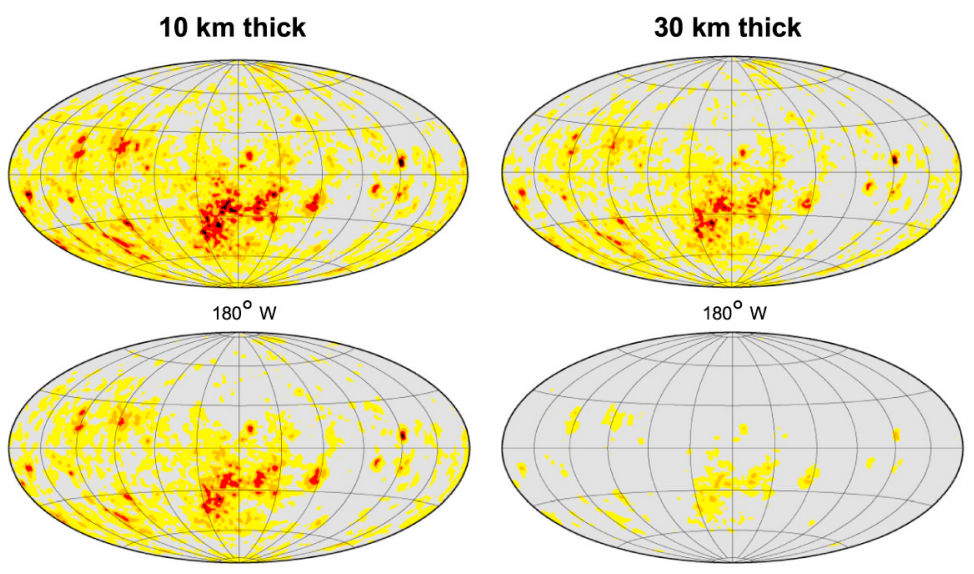

$180^{\circ} \mathrm{W}$
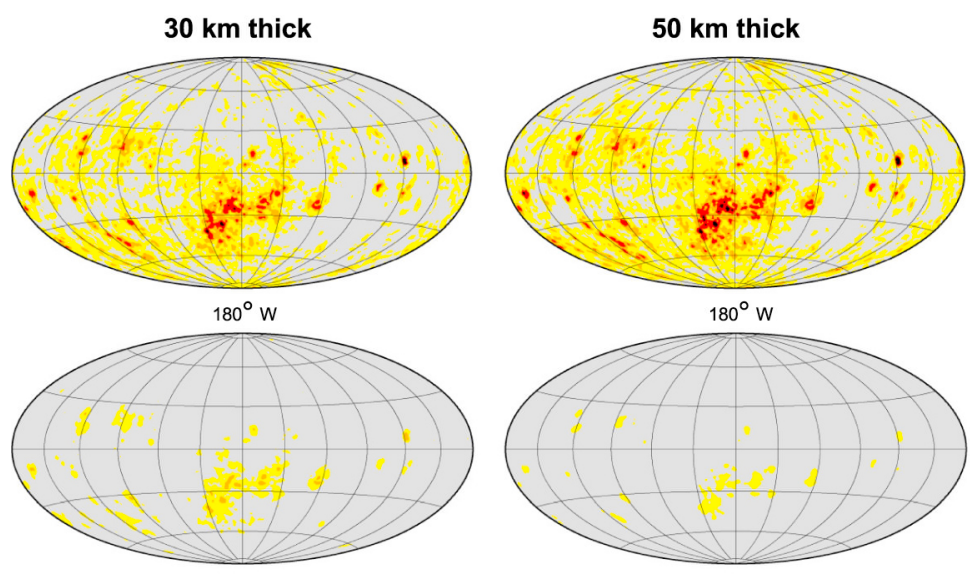

$180^{\circ} \mathrm{W}$

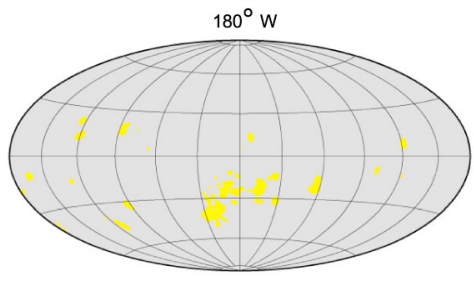

Magnetization $(\mathrm{mA} / \mathrm{m})$

Figure 1. The total magnetizations at (top) the surface and (bottom) base of the magnetized layer for models with magnetized layers of (a) $10 \mathrm{~km}$ thickness with $\lambda=15,000$, (b) $30 \mathrm{~km}$ thickness with $\lambda=20,000$, and (c) $50 \mathrm{~km}$ thickness with $\lambda=16,000$, from three components of the magnetic field synthesized from the spherical harmonic model of Purucker [2008] (Lambert projection centered on the far side, $0.5^{\circ}$ gridded points). 


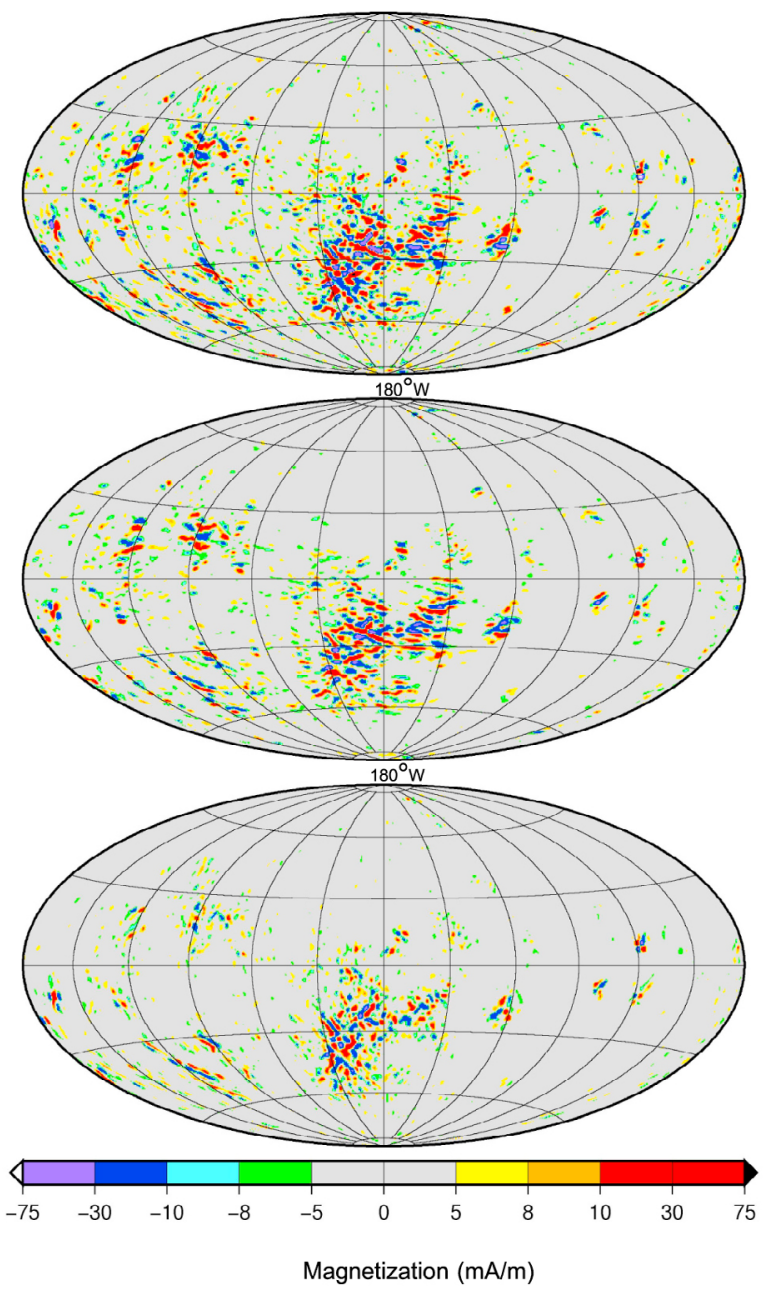

$M_{r}$

$\mathbf{M}_{\mathbf{t}}$

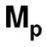

Figure 2. Three component magnetizations at the surface for the global magnetization model (with a $30 \mathrm{~km}$ thick layer and $\lambda=20,000$ ) from three components of the magnetic field synthesized from the spherical harmonic model of Purucker [2008] (Lambert projection centered on the far side, $0.5^{\circ}$ gridded points).

radial data), occur where the measured field is maximum, and for large damping parameters.

[32] Power spectra of the radial component of the magnetic field predictions (Figure 4) are weaker than the power of the input field, showing the reduced magnitudes. The resulting spectra for predictions from the selected model with damping parameter 20,000, and an 'over-damped' model $(\lambda=50,000)$ show the good correlation between the predictions from these magnetization models and the input data up to about harmonic degree 100. The decrease in power at higher harmonic degrees results from the use of the damping parameter in the inversion. A similar fall off in power for predictions from highly damped models is seen for the predictions from the models with the radial data, where higher damping parameters were used.

[33] The preferred models with $10 \mathrm{~km}, 30 \mathrm{~km}$ and $50 \mathrm{~km}$ thick magnetized layers have very similar global distributions of residuals. However, there are more small residuals in the model with $10 \mathrm{~km}$ thickness, and larger residuals in the model with $50 \mathrm{~km}$ thickness, despite these models having the same total misfit. The $10 \mathrm{~km}$ thick model fits some of the strong anomalies better than the other thicknesses, though other regions showed very little variation in residual distribution with model thickness.

[34] When used to predict the surface magnetic fields, the models from the radial component data, and the $10 \mathrm{~km}$ thick magnetized layer from the synthesized data, gave slightly better fits to the ER data (Figure 5 for $10 \mathrm{~km}$ thick layer). The predictions and the ER data agree well spatially, with models of all magnetized layer thicknesses giving the same correlation coefficient with the ER data (to 3 significant figures) of 0.639. However, the actual agreement of the ER and predictions varies for each region, with the strongest total surface fields being under-predicted, and the weaker field region values being over-predicted. The largest

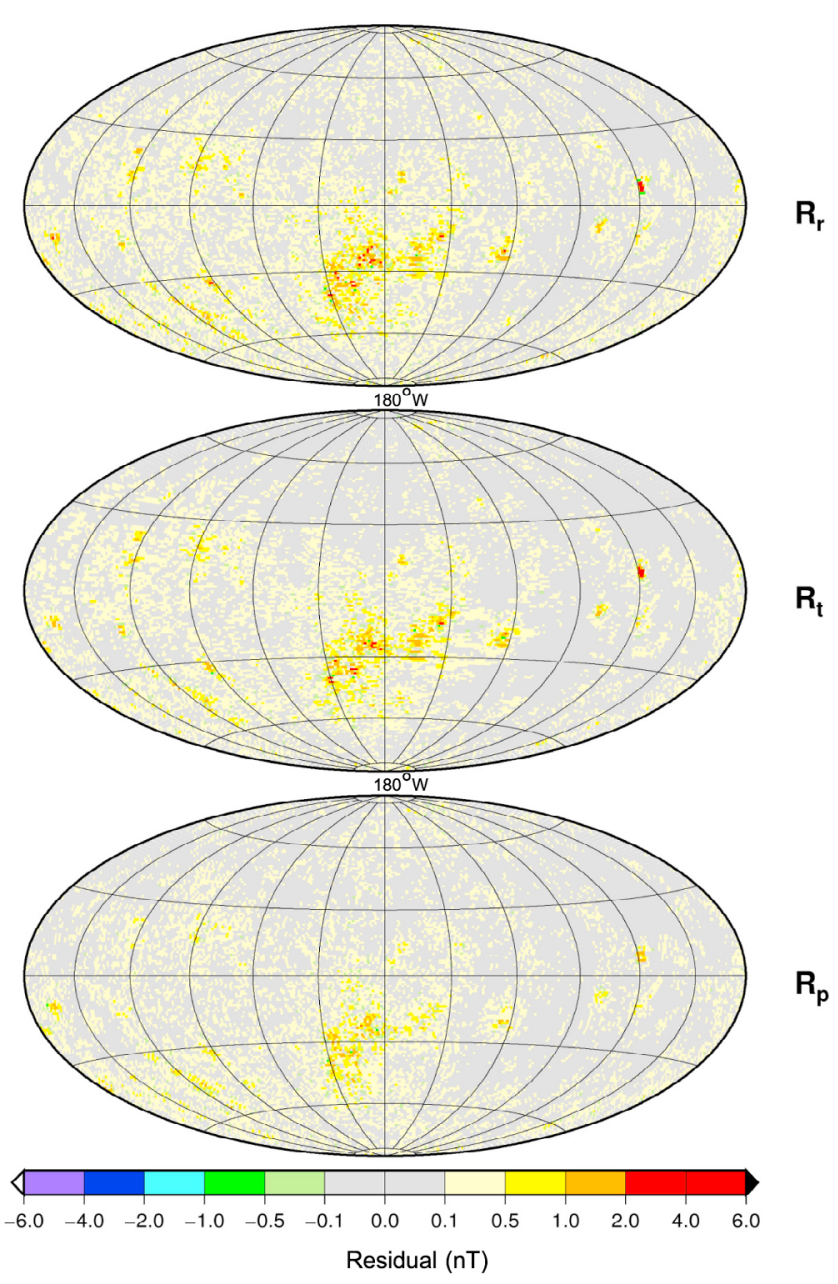

Figure 3. Absolute residuals for the three components of magnetic field from the selected global magnetization model with $30 \mathrm{~km}$ thickness. Positive residuals signify under-prediction, negative residuals signify over-prediction of the input data. (Lambert projection centered on the far side, $1^{\circ}$ gridded points). 


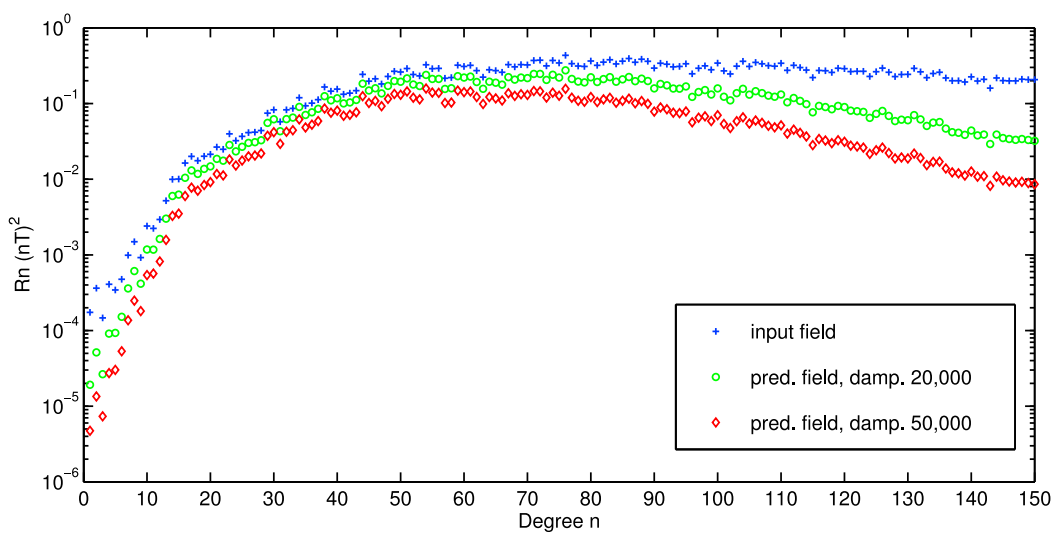

Figure 4. Comparison of the spatial magnetic power spectra, $\mathrm{Rn}$ (at the surface), of the synthetic radial data (blue crosses) [Purucker, 2008] and radial predictions from the selected model with $\lambda=20,000$ (green circles) and an 'over-damped' model ( $\lambda=50,000$, red diamonds).

differences between the data and predictions occur in the regions of very strong $(>10 \mathrm{~s} \mathrm{nT})$ or very weak $(<$ few $\mathrm{nT})$ surface magnetic field.

\section{Discussion}

[35] The magnetization distribution and amplitude remains considerably robust for a range of damping parameters and thicknesses. Magnitudes differ only by a factor of $<4$, allowing constrained estimates of likely lunar crustal magnetization, unlike the spatially continuous models for the Martian crust which saw the RMS magnetization vary by a factor of 20 for models with misfits different by only a factor of 2 [Whaler and Purucker, 2005]. An upper value of the intrinsic model uncertainty in the magnetization values in our models can be estimated from the differences between the magnetization solutions produced when the radial input data grid was shifted. For the Reiner Gamma region, the maximum differences are $20-25 \mathrm{~mA} / \mathrm{m}$ for the largest values of magnetization. While this can be more than $100 \%$ of the magnetizations at some locations, such large differences result from the slight shifting of the resulting magnetization distribution with each input data set, and the mean difference of $\sim 4 \mathrm{~mA} / \mathrm{m}$, for regions with magnetization $>5 \mathrm{~mA} / \mathrm{m}$, is a better estimate of the model uncertainty.

[36] There is 'one to one' correspondence of the directions of magnetization and the vector components of the crustal field, despite the inversion not fixing source locations, meaning that the magnetization directions in these models are unlikely to be representative of true lunar magnetization directions. The horizontal extent of the sources, though coincident with the magnetic field feature, may be more compact than these magnetization models show due to the horizontal spreading of the magnetization solution across the modeled layer. As a result, there is no additional information about signatures associated with particular lunar anomalies.

[37] An inversion technique minimizing spatial variation rather than amplitude may have resulted in a solution with less directional variation. The magnetization modeling method used here however, is not conducive to producing large regions with uniform magnetization direction, so calculation of the magnetization inclination and declination is not meaningful. In addition, care is required in the interpretation of the magnetized sources in some regions where linear looking features in one or more magnetization component may not be linear when the total magnetization, or another magnetization component is considered. Such correspondence between the components of magnetization and the components of the field is also observed in crustal magnetization models at Mars and the Earth [Whaler and Purucker, 2005, 2010].

[38] While magnetization directions from these models are to be treated with caution, the magnetization strengths appear to be reliable. The magnetization strengths of these
ER surface

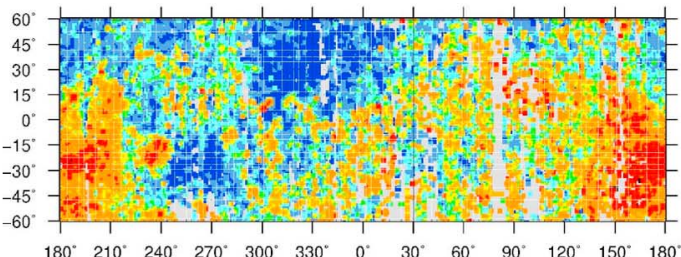

Predicted at surface

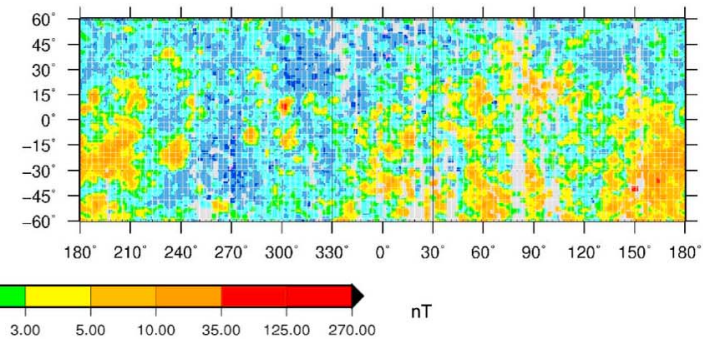

Figure 5. Comparison of (left) the mid latitude $1^{\circ}$ binned ER data with (right) the surface total field predictions at the same locations from the global model with a $10 \mathrm{~km}$ thick layer and $\lambda=15,000$. 
models satisfy the analytical bound to account for the observed magnetic fields provided from ideal body theory [Parker, 2003] and are consistent with measured and estimated values of lunar magnetization. Magnetization values of $0.5-200 \mathrm{~mA} / \mathrm{m}$, as mass normalized dipole moments (for magnetizations on a grid with $0.25^{\circ}$ spacing) are $\sim 4 \times 10^{-8}-1 \times 10^{-4} \mathrm{Am}^{2} \mathrm{~kg}^{-1}$, comparable to the remanent magnetizations measured for the Apollo samples at $10^{-7}-10^{-1} \mathrm{Am}^{2} \mathrm{~kg}^{-1}$ [Fuller and Cisowski, 1987], and differentiated meteorites $\left(\sim 10^{-3} \mathrm{Am}^{2} \mathrm{~kg}^{-1}\right)$ [Kivelson et al., 1993]. Mitchell et al. [2008] estimated the magnetization of a uniformly magnetized impact melt in the Imbrium basin based on the weak fringing fields of craters within this basin at $10^{-6} \mathrm{Am}^{2} \mathrm{~kg}^{-1}$, and this model shows similar magnitudes in that region.

[39] The minimization of the solution norm means that these magnetization magnitudes are minimum estimates of lunar crustal magnetization. Like the magnitudes of the crustal fields, the lunar crustal magnetizations are very much weaker than those on Earth and Mars for layers of comparable thickness. In fact, the highest magnetizations on the Moon of $\sim 0.2 \mathrm{~A} / \mathrm{m}$ are barely significant compared to typical magnetizations observed on Earth of 1-5 A/m [Wasilewski and Mayhew, 1982]. While thinner layers and smaller scale features are likely to have much stronger magnetizations than the maximum values obtained here for a layer with constant global thickness, lunar magnetizations are unlikely to be comparable in magnitude to those on Earth and Mars due to the differences in magnetic mineralogy, the strength of the magnetizing fields, and the processes of magnetization.

[40] The global misfits for these models are within the noise levels of the input data, and the difference in misfits for models with magnetized layers in the range 10-50 km thick are small; thus there is no clear preferred layer thickness. However, the smaller misfits and RMS magnetizations for the model with a $30 \mathrm{~km}$ thick layer, and the persistence of magnetization features at depths greater than $10 \mathrm{~km}$ but less than $50 \mathrm{~km}$ (Figure 1), suggests that globally the magnetized sources of the lunar crust can be represented by a layer $\sim 30 \mathrm{~km}$ thick. It is also possible to estimate a magnetic layer thickness using Parker's [2003] ideal body method in a reverse sense; if the magnetizations are the minimum required to give the fields at altitude, Parker's [2003] expressions show that layers could be as thin as $3 \mathrm{~km}$ for the strongest magnetizations, but the average magnetizations give thicknesses of $10-15 \mathrm{~km}$. It is not expected that all the magnetized regions will have the same thickness, and there are likely to be regions over the globe which can be better described by thinner or thicker layers of magnetized crust (for example the thicker crust of the lunar farside). Indeed, the residuals over some regions, and the agreement of predictions with surface field estimates, are improved slightly if a thinner magnetized layer $(\sim 10 \mathrm{~km})$ is used.

[41] The disagreement with some of the surface data results from the difference in sensitivity of the MAG and the ER technique to sources with different wavelengths, and the use of the damping parameter in these inversions. The strongest surface features are under-predicted by the model, as the ER technique is more sensitive to smaller wavelength sources than the MAG. Over-prediction of the weaker surface fields is also observed with the spherical harmonic model, and may occur as a result of the amplification of noise in the MAG data when effectively downward continued to the surface, or demonstrate the variation in the response of the ER technique to the very small wavelength features anticipated from the Apollo surface data.

\section{Conclusions}

[42] Spatially continuous global crustal magnetization models for the lunar crust have been produced using the method of Whaler and Purucker [2005]. These are the first global models of lunar crustal magnetization, and have magnetization solutions with minimum RMS magnetization amplitude for a given fit to the data, controlled by a damping parameter. Models were produced from both the radial component of the MAG data from the wake and tail regions, and from the three components of the magnetic field synthesized from a degree 150 spherical harmonic model of the lunar crustal field. The resulting distributions of crustal magnetization produced from both data sets show similar patterns, demonstrating robustness. The magnetization models can account for many of the features of the observed magnetic fields at altitude, and for some of the surface fields as measured with the ER, though amplitudes are often not reproducible.

[43] Surface predictions from these magnetization models provide a better prediction of some of the weaker surface fields than the degree 150 spherical harmonic model. However, as a single layer thickness globally will not be appropriate everywhere, and a single choice of model will fit individual data points differently, predictions from this magnetization model fail to capture some of the field information, particularly on small spatial scales (higher harmonic degrees), contained in the unmodeled data or spherical harmonic model.

[44] The suggested $30 \mathrm{~km}$ thick magnetized layer is consistent with the spectral thickness calculated, and represents a global compromise of the fit to the data and RMS magnetization. For example, thinner layers provided a better fit in the Reiner Gamma region, compatible with theories that the magnetized source in this region is a thin near-surface layer of impact ejecta [Hood et al., 2001; Nicholas et al., 2007] (though the layer would likely be thinner than the thinnest modeled here). Models with layer thicknesses $>10 \mathrm{~km}$ require magnetization magnitudes that are comparable to those measured in the Apollo samples, demonstrating that minimum magnetization models from satellite data provide useful bounds on the magnetization of the lunar crust, and suggest that the magnetic mineralogies of the Apollo samples are representative of the lunar crust as a whole.

[45] The magnetization strengths suggest that magnetizing fields from a few $\mu \mathrm{T}$ (as deduced for some Apollo samples) to more than $40 \mu \mathrm{T}$ (for shock magnetization [Gattacceca et al., 2010]) might have been present on the Moon. The persistence of these magnetization strengths at depth $(>10 \mathrm{~km})$ in some regions requires the magnetizing field to exist in, or be able to penetrate into, the lunar crust, in addition to the mechanism for producing the remanence. As transient fields generated in impacts are expected to penetrate into the lunar crust [Hood and Artemieva, 2008], magnetization at depth 
does not distinguish impact generated fields from a dynamo field unless magnetization was acquired during cooling, requiring a field steady over long periods of time. However, in the absence of field amplification, a dynamo field may not be strong enough to explain some of the stronger magnetizations, and thus a combination of different fields and mechanisms may be needed to explain the lunar crustal fields.

[46] While this modeling method did not permit magnetization directions to be determined, some of the spatially rapid directional variation in these magnetization models may be real features of lunar crustal magnetization, suggesting that the directions of the magnetizing fields may have varied on small spatial scales. Such small scale variation within a magnetizing field is more likely to be associated with impact generated magnetic fields than a dynamo field.

[47] With the excellent coverage of the LP (and now also Kaguya) MAG data [e.g., Tsunakawa et al., 2010], and new models of the internal magnetic field [Purucker and Nicholas, 2010], it is not necessarily the resolution or coverage of the crustal field measurements which are limiting the conclusions on the sources of lunar crustal magnetism (though surface surveys such as that from a lunar rover would of course provide valuable information); rather, as in the terrestrial case also, it is the deciphering of the exact sources of the fields which is still lacking.

[48] Ultimately, a better understanding of the magnetization of the lunar crust and the sources themselves will come from regional magnetization models, more lunar samples, and new geophysical data. Ground truth magnetization directions and rock compositions for regions like Reiner Gamma and impact antipodes, coupled with, for example, seismic and/or radar surveys, may constrain the true sources of these anomalies and their magnetizing fields. Re-analysis of the magnetic properties of the Apollo samples, and experimental studies of shock remanent magnetization [e.g., Gattacceca et al., 2007, 2008, 2010] is ongoing to improve the understanding of the magnetic and paleomagnetic properties of the lunar rocks.

\section{Appendix A}

[49] Voorhies et al. [2002] used the theoretical power spectrum expected from a large number of uncorrelated dipoles, with random magnetic moments, scattered on a sphere within a spherical body to find the best fitting source radius for different sections of the observed power spectra for the crustal field of Earth and Mars. Applying this technique to the Moon, the best fitting power spectrum for degrees 1 to 150 is shown in Figure A1 with the observed power spectrum. The higher degree terms fit the theoretical model better than the lower degree terms, which are perhaps dominated by noise from unmodeled external fields.

[50] The best fitting source sphere radius of $1711.4 \mathrm{~km}$ gives a globally averaged source depth of $25.7 \mathrm{~km}$. The globally averaged source depth varies from $\sim 14$ to $30 \mathrm{~km}$ depending on which parts of the spectrum are fit. The estimated source depth from this technique is considered to be closer to the layer thickness than the layer half thickness as theoretical spectra may overestimate the true depth of sources that are correlated over scales larger than the dipole

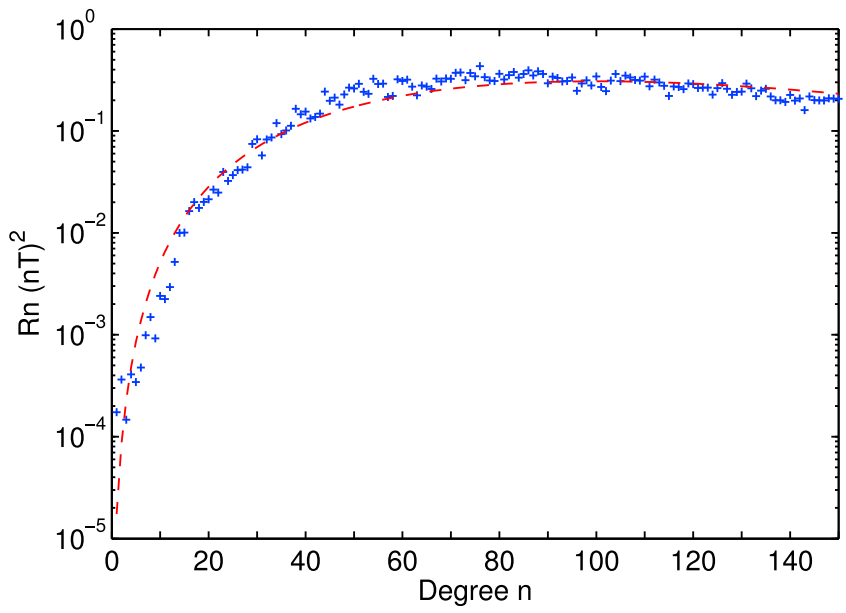

Figure A1. The spatial magnetic power spectra, Rn, for the lunar crustal field up to degree 150 at the surface (blue crosses) with a theoretical curve fitted through it (red line) expected from a spherical shell of randomly oriented dipoles.

spacing [Voorhies, 2008]. Although this 'spectral thickness' does not take into account any correlation of the sources, or variations in layer thickness which would be expected for some regions of the Moon, a layer thickness of 25-30 km serves as a first estimate for the global average magnetic crustal thickness of the Moon.

[51] Acknowledgments. This research was funded by the Natural Environment Research Council (NER/S/A/2006/14230). Maps are plotted using Generic Mapping Tools [Wessel and Smith, 1998]. We would like to thank Benoit Langlais, Mark Wieczorek and two anonymous reviewers for their helpful and insightful suggestions.

\section{References}

Binder, A. B. (1998), Lunar Prospector: Overview, Science, 281, 1475-1476, doi:10.1126/science. 281.5382 .1475$.

Collinson, D. W. (1993), Magnetism of the Moon-A lunar core dynamo or impact magnetization?, Surv. Geophys., 14(1), 89-118, doi:10.1007/ BF01044078.

Constable, S. C., R. L. Parker, and C. G. Constable (1987), Occam's Inversion: A practical algorithm for generating smooth models from electromagnetic sounding data, Geophysics, 52(3), 289-300.

Crawford, D. A., and P. H. Schultz (1988), Laboratory observations of impact-generated magnetic fields, Nature, 336, 50-52, doi:10.1038/ $336050 \mathrm{a} 0$.

Dwyer, C. A., D. J. Stevenson, and F. Nimmo (2011), A long-lived lunar dynamo driven by continuous mechanical stirring, Nature, 479, 212-214, doi:10.1038/nature10564.

Dyal, P., C. W. Parkin, and C. P. Sonett (1970), Apollo 12 magnetometer: Measurement of a steady magnetic field on the surface of the Moon, Science, 169, 762-764, doi:10.1126/science.169.3947.762.

Dyal, P., C. W. Parkin, and W. D. Daily (1974), Magnetism and the interior of the Moon, Rev. Geophys., 12, 568-591, doi:10.1029/ RG012i004p00568.

Frey, S., J. S. Halekas, D. L. Mitchell, and R. P. Lin (2002), Cluster analysis of lunar magnetism, gravity anomalies, and topology, paper presented at 33rd Lunar and Planetary Science Conference, Lunar and Planet. Inst., Houston, Tex., 11-15 March.

Fuller, M. (1974), Lunar magnetism, Rev. Geophys., 12, 23-70, doi:10.1029/RG012i001p00023.

Fuller, M., and S. M. Cisowski (1987), Lunar paleomagnetism, in Geomagnetism, vol. 2, edited by J. A. Jacobs, pp. 307-455, Academic, San Diego, Calif.

Garrick-Bethell, I., B. P. Weiss, D. L. Shuster, and J. Buz (2009), Early lunar magnetism, Science, 323(5912), 356-359.

Gattacceca, J., A. Lamali, P. Rochette, M. Boustie, and L. Berthe (2007), The effects of explosive-driven shocks on the natural remanent 
magnetization and the magnetic properties of rocks, Phys. Earth Planet. Inter., 162, 85-98, doi:10.1016/j.pepi.2007.03.006.

Gattacceca, J., L. Berthe, M. Boustie, F. Vadeboin, P. Rochette, and T. de Resseguier (2008), On the efficiency of shock magnetization processes, Phys. Earth Planet. Inter., 166, 1-10, doi:10.1016/j.pepi.2007. 09.005 .

Gattacceca, J., M. Boustie, L. Hood, J.-P. Cuq-Lelandais, M. Fuller, N. S. Bezaeva, T. de Resseguier, and L. Berthe (2010), Can the lunar crust be magnetized by shock: Experimental groundtruth, Earth Planet. Sci. Lett., 299, 42-53, doi:10.1016/j.eps1.2010.08.011.

Halekas, J. S., D. L. Mitchell, R. P. Lin, S. Frey, L. L. Hood, M. H. Acuña, and A. B. Binder (2001), Mapping of crustal magnetic anomalies on the lunar near side by the Lunar Prospector electron reflectometer, J. Geophys. Res., 106(E11), 27,841-27,852, doi:10.1029/2000JE001380.

Halekas, J. S., D. L. Mitchell, R. P. Lin, L. L. Hood, M. H. Acuña, and A. B. Binder (2002), Demagnetization signatures of lunar impact craters, Geophys. Res. Lett., 29(13), 1645, doi:10.1029/2001GL013924.

Halekas, J. S., R. P. Lin, and D. L. Mitchell (2003), Magnetic fields of lunar multi-ring impact basins, Meteorit. Planet. Sci., 38(4), 565-578, doi:10.1111/j.1945-5100.2003.tb00027.x.

Hood, L. L. (1987), Magnetic field and remanent magnetization effects of basin-forming impacts on the Moon, Geophys. Res. Lett., 14, 844-847, doi:10.1029/GL014i008p00844

Hood, L. L. (2011), Central magnetic anomalies of Nectarian-aged lunar impact basins: Probable evidence for an early core dynamo, Icarus, 211(2), $1109-1128$.

Hood, L. L., and N. A. Artemieva (2008), Antipodal effects of lunar basinforming impacts: Initial 3D simulations and comparisons with observations, Icarus, 193(2), 485-502.

Hood, L. L., and Z. Huang (1991), Formation of magnetic anomalies antipodal to lunar impact basins: Two-dimensional model calculations, J. Geophys. Res., 96, 9837-9846, doi:10.1029/91JB00308.

Hood, L. L., and G. Schubert (1980), Lunar magnetic anomalies and surface optical properties, Science, 208, 49-51, doi:10.1126/science.208.4439.49.

Hood, L. L., and A. Vickery (1984), Magnetic field amplification and generation in hypervelocity meteoroid impacts with application to lunar paleomagnetism, J. Geophys. Res., 89, C211-C223, doi:10.1029/ JB089iS01p0C211

Hood, L. L., A. Zakharian, J. S. Halekas, D. L. Mitchell, R. P. Lin, M. H. Acuña, and A. B. Binder (2001), Initial mapping and interpretation of lunar crustal magnetic anomalies using Lunar Prospector magnetometer data, J. Geophys. Res., 106(E11), 27,825-27,839, doi:10.1029/ 2000JE001366.

Housley, R. M., R. W. Grant, and N. E. Paton (1973), Origin and Characteristics of Fe metal in lunar glass welded aggregates, Proc. Lunar Planet Sci. Conf., 4th, 3, 2737-2749.

Jackson, A. (1990), Accounting for crustal magnetization in models of the core magnetic field, Geophys. J. Int., 103, 657-673, doi:10.1111/ j.1365-246X.1990.tb05678.x.

Katanforoush, A., and M. Shahshahani (2003), Distributing points on a sphere I, Expo. Math., 12(2), 119.

Kivelson, M. G., L. F. Bargatze, K. K. Khurana, D. J. Southwood, R. J. Walker, and P. J. Coleman (1993), Magnetic field signatures near Galileo's closest approach to Gaspra, Science, 261(5119), 331-334.

Le Bars, M., M. A. Wieczorek, Ö. Karatekin, D. Cébron, and M. Laneuville (2011), An impact-driven dynamo for the early Moon, Nature, 479, 215-218, doi:10.1038/nature 10565

Lin, R. P., K. A. Anderson, and L. L. Hood (1988), Lunar surface magnetic field concentrations antipodal to young large impact basins, Icarus, 74, 529-541, doi:10.1016/0019-1035(88)90119-4.

Maus, S., and V. Haak (2003), Magnetic field annihilators: Invisible magnetization at the magnetic equator, Geophys. J. Int., 155, 509-513, doi:10.1046/j.1365-246X.2003.02053.x.

Mitchell, D. L., J. S. Halekas, R. P. Lin, S. Frey, L. L. Hood, M. H. Acuña, and A. Binder (2008), Global mapping of lunar crustal magnetic fields by Lunar Prospector, Icarus, 194(2), 401-409, doi:10.1016/j.icarus.2007. 10.027

Nicholas, J. B., M. E. Purucker, and T. J. Sabaka (2007), Age spot or youthful marking: Origin of Reiner Gamma, Geophys. Res. Lett., 34, L02205, doi:10.1029/2006GL027794.

Parker, R. L. (2003), Ideal bodies for Mars magnetics, J. Geophys. Res., 108(E1), 5006, doi:10.1029/2001JE001760.
Parker, R. L., L. Shure, and J. Hildebrand (1987), The application of inverse theory to seamount magnetism, Rev. Geophys., 25, 17-40, doi:10.1029/ RG025i001p00017.

Press, W. H., S. A. Teukolsky, W. T. Vetterling, and B. P. Flannery (1992), Numerical Recipes in Fortran, 2nd ed., Cambridge Univ. Press, Cambridge, U. K.

Purucker, M. E. (2008), A global model of the internal magnetic field of the Moon based on Lunar Prospector magnetometer observations, Icarus, 197(1), 19-23, doi:10.1016/j.icarus.2008.03.016.

Purucker, M. E., and J. B. Nicholas (2010), Global spherical harmonic models of the internal magnetic field of the Moon based on sequential and coestimation approaches, J. Geophys. Res., 115, E12007, doi:10.1029/2010JE003650.

Purucker, M. E., T. J. Sabaka, and R. A. Langel (1996), Conjugate gradient analysis: A new tool for studying satellite magnetic data sets, Geophys. Res. Lett., 23, 507-510, doi:10.1029/96GL00388.

Purucker, M. E., J. W. Head, and J. S. Halekas (2008), Magnetic signatures of lunar multi-ringed impact basins: New constraints on the timing of the putative lunar dynamo, paper presented at 39th Lunar and Planetary Science Conference, Lunar and Planet. Inst., League City, Tex., 10-14 March.

Richmond, N. C., and L. L. Hood (2008), A preliminary global map of the vector lunar crustal magnetic field based on Lunar Prospector magnetometer data, J. Geophys. Res., 113, E02010, doi:10.1029/2007JE002933.

Richmond, N. C., L. L. Hood, J. S. Halekas, D. L. Mitchell, R. P. Lin, M. H. Acuña, and A. B. Binder (2003), Correlation of a strong lunar magnetic anomaly with a high-albedo region of the Descartes mountains, Geophys. Res. Lett., 30(7), 1395, doi:10.1029/2003GL016938.

Runcorn, S. K. (1975), On the interpretation of Lunar magnetism, Phys. Earth Planet. Inter, 10, 327-335, doi:10.1016/0031-9201(75)90059-X.

Runcorn, S. K., D. W. Collinson, W. O'Reilly, A. Stephenson, N. N. Greenwood, and M. H. Battey (1970), Magnetic properties of lunar samples, Science, 167(3918), 697-699.

Shure, L., R. L. Parker, and G. E. Backus (1982), Harmonic splines for geomagnetic modeling, Phys. Earth Planet. Inter., 28, 215-229, doi:10.1016/0031-9201(82)90003-6.

Srnka, L. J. (1977), Spontaneous magnetic field generation in hypervelocity impacts, Proc. Lunar Planet. Sci. Conf., 8th, 1, 792-795.

Stegman, D. R., A. M. Jellinek, S. A. Zatman, J. R. Baumgarder, and M. A. Richards (2003), An early lunar core dynamo driven by thermochemical mantle convection, Nature, 421, 143-146, doi:10.1038/ nature 01267.

Strangway, D. W., W. Gose, and R. K. McConnell (1973), Lunar magnetic anomalies and the Cayley Formation, Nature, 246, 112-114.

Tsunakawa, H., H. Shibuya, F. Takahashi, H. Shimizu, M. Matsushima A. Matsuoka, S. Nakazawa, H. Otake, and Y. Iijima (2010), Lunar Magnetic Field Observation and Initial Global Mapping of Lunar Magnetic Anomalies by MAP-LMAG Onboard SELENE (Kaguya), Space Sci. Rev., 154, 219-251.

Voorhies, C. V. (2008), Thickness of the magnetic crust of Mars, J. Geophys. Res., 113, E04004, doi:10.1029/2007JE002928.

Voorhies, C. V., T. J. Sabaka, and M. E. Purucker (2002), On magnetic spectra of Earth and Mars, J. Geophys. Res., 107(E6), 5034, doi:10.1029/ 2001JE001534.

Wasilewski, P., and M. A. Mayhew. (1982) Crustal xenolith magnetic properties and long wavelength anomaly source requirements, Geophys. Res. Lett., 9(4), 329-332, doi:10.1029/GL009i004p00329.

Wessel, P., and W. H. F. Smith (1998), New, improved version of generic mapping tools released, Eos Trans. $A G U, 79(47)$, 579, doi:10.1029/ 98EO00426.

Whaler, K. A., and R. A. Langel (1996), Minimal crustal magnetizations from satellite data, Phys. Earth Planet. Inter., 98, 303-319, doi:10.1016/S0031-9201(96)03193-7.

Whaler, K. A., and M. E. Purucker (2005), A spatially continuous magnetization model for Mars, J. Geophys. Res., 110, E09001, doi:10.1029/ 2004JE002393.

Whaler, K. A., and M. E. Purucker (2010), Magnetization models derived from high resolution satellite lithospheric anomaly fields, paper presented at ESA's Second Swarm International Science Meeting, GFZ, Potsdam, Germany.

Wieczorek, M. A et al. (2006), The constitution and structure of the lunar interior, in New Views of the Moon, edited by B. L. Jolliff et al., Rev. Mineral. Geochem., 60, 221-364, doi:10.2138/rmg.2006.60.3. 